\title{
Fast Numerical Method for Pricing of Variable Annuities with Guaranteed Minimum Withdrawal Benefit under Optimal Withdrawal Strategy
}

\author{
Xiaolin Luo ${ }^{1, *}$ and Pavel V. Shevchenko ${ }^{2}$ \\ Draft, this version 24 October 2014 \\ 1 The Commonwealth Scientific and Industrial Research Organisation, Australia; e-mail: \\ Xiaolin.Luo@csiro.au \\ 2 The Commonwealth Scientific and Industrial Research Organisation, Australia; e-mail: \\ Pavel.Shevchenko@csiro.au \\ * Corresponding author
}

\begin{abstract}
A variable annuity contract with Guaranteed Minimum Withdrawal Benefit (GMWB) promises to return the entire initial investment through cash withdrawals during the policy life plus the remaining account balance at maturity, regardless of the portfolio performance. Under the optimal withdrawal strategy of a policyholder, the pricing of variable annuities with GMWB becomes an optimal stochastic control problem. So far in the literature these contracts have only been evaluated by solving partial differential equations (PDE) using the finite difference method. The well-known Least-Squares or similar Monte Carlo methods cannot be applied to pricing these contracts because the paths of the underlying wealth process are affected by optimal cash withdrawals (control variables) and thus cannot be simulated forward in time. In this paper we present a very efficient new algorithm for pricing these contracts in the case when transition density of the underlying asset between withdrawal dates or its moments are known. This algorithm relies on computing the expected contract value through a high order Gauss-Hermite quadrature applied on a cubic spline interpolation. Numerical results from the new algorithm for a series of GMWB contract are then presented, in comparison with results using the finite difference method solving corresponding PDE. The comparison demonstrates that the new algorithm produces results in very close agreement with those of the finite difference method, but at the same time it is significantly faster; virtually instant results on a standard desktop PC.
\end{abstract}

Keywords: Variable Annuity, Optimal Stochastic Control, Guaranteed Minimum Withdrawal Benefit, Gauss-Hermite Quadrature, Cubic Spline. 


\section{Introduction}

The world population is becoming older fast. According to world population data recently published in United Nations (2013) there were about 800 million persons aged 60 years or over in 2012, but this number is expected to grow to 2 billion by 2050. The oldest old (aged 80 or over) population accounted for $14 \%$ of the older population (aged over 60 ) and this is expected to grow to $20 \%$ by 2050 . The number of of centenarians (aged 100 years or over) is growing even faster. It is projected to increase tenfold, from approximately 343,000 in 2012 to 3.2 million by 2050. The old-age support ratio (number of persons aged 15 to 64 years per person aged 65 years or over) is 8 to 1 now, but it will be reduced to 4 to 1 by 2050. As a consequence the age-related spending is projected to rise dramatically in the coming decades in all the developed countries. Increasingly governments in the developed world realize they cannot afford paying sufficient public pensions and are looking for innovations in the financial market for providing some urgently needed solutions. Variable annuity is one of the products that can help with meeting the challenges posed by the so called longevity risk.

In this paper we consider a variable annuity contract with Guaranteed Minimum Withdrawal Benefit (GMWB) that promises to return the entire initial investment through cash withdrawals during the policy life plus the remaining account balance at maturity, regardless of the portfolio performance. Thus even when the account of the policyholder falls to zero before maturity, GMWB feature will continue to provide the guaranteed cashflows. GMWB allows the policyholder to withdraw funds below or at contractual rate without penalty and above the contractual rate with some penalty. If the policyholder behaves passively and the withdraw amount at each withdrawal date is predetermined at the beginning of the contract, then the behavior of the policyholder is called "static". In this case the paths of the account can be simulated and a standard Monte Carlo simulation method can be used to price the GMWB. On the other hand if the policyholder optimally decide the amount of withdraw at each withdrawal date, then the behavior of the policyholder is called "dynamic". A rational policyholder of GMWB will always choose the optimal withdrawal strategy to maximize the present value of cash flows generated from holding the GMWB. Under the optimal withdrawal strategy of a policyholder, the pricing of variable annuities with GMWB becomes an optimal stochastic control problem. This problem cannot be solved by a simulation based method such as the well known Least-Squares Monte Carlo method introduced in Longstaff and Schwartz (2001), due to the fact that the paths of the underlying wealth process are altered by the optimal cash withdrawals that should be found from backward solution and the underlying wealth process cannot be simulated forward in time. 
The variable annuities with GMWB feature have been considered in a number of papers over the last decade. Milevsky and Salisbury (2006) developed a variety of methods for pricing GMWB products. In their static approach the GMWB product is decomposed into a Quanto Asian put plus a generic term-certain annuity. In their dynamic approach they assume the policyholder can terminate (surrender) the contract at the optimal time, which leads to an optimal stopping problem akin to pricing an American put option. Bauer et al. (2008) presents valuation of variable annuities with multiple guarantees. In their dynamic approach a strategy not only consists of the decision whether or not to surrender, but also a numerous possible withdrawal amounts at each payment date. They have developed a multidimensional discretization approach in which the Black-Scholes PDE is transformed to a one-dimensional heat equation and a quasi-analytic solution is obtained through a simple piecewise summation with a linear interpolation on a mesh. Unfortunately the numerical formulation considered in Bauer et al. (2008) has four dimensions and the computation of even a single price of the GMWB contract under the optimal policyholder strategy is very costly; it is mentioned in their paper that it took more than 15 hours CPU on an Intel Pentium IV with $2.80 \mathrm{GHz}$ and $1 \mathrm{~GB}$ RAM, and no results for the dynamic case were shown. Dai et al. (2008) developed an efficient finite difference algorithm using the penalty approximation to solve the singular stochastic control problem for a continuous withdrawal model under the dynamic (optimal) withdrawal strategy. They have also developed a finite difference algorithm for the more realistic discrete withdrawal formulation. Their results show that the GMWB values from the discrete model converge to those of the continuous model. Chen and Forsyth (2008) present an impulse stochastic control formulation for pricing variable annuities with GMWB under the optimal policyholder behavior, and develop a single numerical scheme for solving the Hamilton-Jacobi-Bellman variational inequality for the continuous withdrawal model as well as for pricing the discrete withdrawal contracts. In Bacinello et al. (2011) the static valuations are performed via ordinary Monte Carlo method, and the mixed valuations, where the policyholder is 'semiactive' and can decide to surrender the contract at any time during the life of the GMWB contract, are performed by the Least-Squares Monte Carlo method.

In the case when transition density of the underlying wealth process between withdrawal dates or its moments are known in closed form, often it can be more convenient and more efficient to utilize direct integration methods to calculate the required option price expectations in backward time-stepping procedure. In this paper we present a method that relies on computing the expected option values in a backward timestepping between withdrawal dates through Gauss-Hermite integration quadrature ap- 
plied on a cubic spline interpolation. For convenience, hereafter we refer this new algorithm as GHQC (Gauss-Hermite quadrature on cubic spline). We adopt the method developed in Luo and Shevchenko (2014) for pricing American options and extend it to solving optimal stochastic control problem for pricing GMWB variable annuity. This allows to get virtually instant results for typical GMWB annuity prices on the standard desktop PC. In this paper we consider pricing variable annuities with GMWB under both static and dynamic (optimal) policyholder behaviors. Here our definition

of "dynamic" is similar to the one used by Bauer et al. (2008), Dai et al. (2008) and Chen and Forsyth (2008), i.e. the rational policyholder can decide an optimal amount to withdraw at each discrete payment date to maximize the expected discounted value of the cash flows generated from holding the variable annuity with GMWB. At discrete withdrawing dates the proper jump conditions are applied at various withdrawal account levels, allowing the optimal withdrawal decision to be made by choosing a withdrawal amount to maximize the cashflow. Once the fair price of GMWB for given inputs is evaluated, the fair fee charged by the policy issuer can be determined iteratively by matching the initial premium and the fair price.

In the next section we describe the GMWB product with discrete withdrawals, the underlying stochastic model and the optimization problem. Section 3 presents the GHQC algorithm for pricing the GMWB contracts under both static and dynamic (optimal) policyholder behaviors. In Section 4, numerical results for the fair fees under a series GMWB contract conditions are presented, in comparison with the results from solving PDEs using finite difference method. The comparison demonstrates that the new algorithm produces results very close to those of the finite difference method, but at the same time it is significantly faster. Concluding remarks are given in Section 5 .

\section{Model}

Consider the GMWB annuity contract and underlying asset stochastic model as follows.

- Let $S(t)$ denote the value of the reference portfolio of assets (mutual fund index, etc.) underlying the variable annuity policy at time $t$ that under no-arbitrage condition follows the risk neutral stochastic process

$$
d S(t)=r(t) S(t) d t+\sigma(t) S(t) d B(t)
$$

where $B(t)$ is the standard Wiener process, $r(t)$ is risk free interest rate and $\sigma(t)$ is volatility. For simplicity hereafter we assume that model parameters are piecewise constant functions of time for time discretization $0=t_{0}<t_{1}<\cdots<t_{N}=T$, where $t_{0}=0$ is today and $T$ is annuity contract maturity. Denote corresponding 
asset values as $S\left(t_{0}\right), S\left(t_{1}\right), \ldots, S\left(t_{N}\right)$; and risk-free interest rate and volatility as $r_{1}, \ldots, r_{N}$ and $\sigma_{1}, \ldots, \sigma_{N}$ respectively. That is $r_{1}$ is the interest rate for time teriod $\left(t_{0}, t_{1}\right] ; r_{2}$ is for $\left(t_{1} ; t_{2}\right]$, etc and similar for volatility.

- The premium paid by policyholder upfront at $t_{0}$ is invested into the reference portfolio of risky assets $S(t)$. Denote the value of this variable annuity account (hereafter referred to as wealth account) at time $t$ as $W(t)$, i.e. the upfront premium paid by policyholder is $W(0)$. GMWB guarantees the return of the premium via withdrawals $\gamma_{n} \geq 0$ allowed at times $t_{n}, n=1,2, \ldots, N$. Let $N_{w}$ denote the number of withdrawals in a year (e.g. $N_{w}=12$ for a monthly withdrawal), then the total number of withdrawals $N=\left\lceil N_{w} \times T\right\rceil$, where $N=\lceil\cdot\rceil$ denotes the ceiling of a float number. The total of withdrawals cannot exceed the guarantee $W(0)$ and withdrawals can be different from contractual (guaranteed) withdrawal $G_{n}=W(0)\left(t_{n}-t_{n-1}\right) / T$, with penalties imposed if $\gamma_{n}>G_{n}$. Denote the annual contractual rate as $g=1 / T$.

- Denote the value of the guarantee at time $t$ as $A(t)$, hereafter referred to as guarantee account. Obviously, $A(0)=W(0)$. For clarity of notation, denote the time immediately before $t$ as $t^{-}$, and immediately after $t$ as $t^{+}$, and functions of time are left continuous, e.g. $A\left(t_{n}\right)=A\left(t_{n}^{+}\right)$etc. Then the guarantee balance evolves as

$$
A\left(t_{n}^{+}\right)=A\left(t_{n}^{-}\right)-\gamma_{n}=A\left(t_{n-1}^{+}\right)-\gamma_{n}, \quad n=1,2, \ldots, N
$$

with $A\left(T^{+}\right)=0$, i.e. $W(0)=A(0) \geq \gamma_{1}+\cdots+\gamma_{N}$ and $A\left(t_{n-1}\right)=A\left(t_{n-1}^{+}\right) \geq$ $\sum_{k=n}^{N} \gamma_{k}$. The account balance $A(t)$ remains unchanged within the interval $\left(t_{n-1}, t_{n}\right), n=1,2, \ldots, N$.

- In the case of reference portfolio process (1), the wealth account $W(t)$ evolves as

$$
W\left(t_{n}\right)=\max \left[W\left(t_{n-1}\right) e^{\left(r_{n}-\alpha-\frac{1}{2} \sigma_{n}^{2}\right) d t_{n}+\sigma_{n} \sqrt{d t_{n}} z_{n}}-\gamma_{n}, 0\right], \quad n=1,2, \ldots, N,
$$

where $d t_{n}=t_{n}-t_{n-1}, z_{n}$ are iid standard Normal random variables and $\alpha$ is the annual fee charged by the insurance company. If the account balance becomes zero or negative, then it will stay zero till maturity. Then from $t=t_{n-1}^{+}$to $t=t_{n}^{-}$ the value of wealth account $W(t)$ evolves as

$$
W\left(t_{n}^{-}\right)=W\left(t_{n-1}^{+}\right) e^{\left(r_{n}-\alpha-\frac{1}{2} \sigma_{n}^{2}\right) d t_{n}+\sigma_{n} \sqrt{d t_{n}} z_{n}}, \quad n=1,2, \ldots, N
$$

Note the process for $W(t)$ within $\left(t_{n-1}, t_{n}\right)$ differs from the process for the underlying asset $S(t)$ only in the drift; the former has a reduced drift due to the continuously charged fee $\alpha$ on the GMWB contract. 
- The cashflow received by the policyholder at withdrawal time $t_{n}$ is given by

$$
C\left(\gamma_{n}\right)= \begin{cases}\gamma_{n}, & \text { if } 0 \leq \gamma_{n} \leq G_{n}, \\ G_{n}+(1-\beta)\left(\gamma_{n}-G_{n}\right), & \text { if } \gamma_{n}>G_{n},\end{cases}
$$

where $G_{n}$ is contractual withdrawal. That is, penalty is applied if withdrawal $\gamma_{n}$ exceeds $G_{n}$, i.e. $\beta \in[0,1]$ is the penalty applied to the portion of withdrawal above $G_{n}$.

- Denote the value of variable annuity at time $t$ as $Q_{t}(W(t), A(t))$, i.e. it is determined by values of the wealth and guarantee accounts $W(t)$ and $A(t)$. At maturity, the policyholder takes the maximum between the remaining guarantee withdrawal net of penalty charge and the remaining balance of the personal account, i.e. the final payoff is

$$
Q_{t_{N}^{-}}\left(W\left(T^{-}\right), A\left(T^{-}\right)\right)=\max \left(W\left(T^{-}\right), C\left(A\left(T^{-}\right)\right)\right) .
$$

The policyholder receives cashflows $C\left(\gamma_{n}\right), \quad n=1,2, \ldots, N-1$ and the final payoff at maturity. The present value of total payoff is

$$
P_{0}=e^{-r_{0, N} T} \max \left(W\left(T^{-}\right), C\left(A\left(T^{-}\right)\right)\right)+\sum_{n=1}^{N-1} e^{-r_{0, n} t_{n}} C\left(\gamma_{n}\right),
$$

where $r_{i, n}=\frac{1}{t_{n}-t_{i}} \int_{t_{i}}^{t_{n}} r(\tau) d \tau, t_{n}>t_{i}$.

Under the above assumptions/conditions, the fair no-arbitrage value of the annuity at time $t_{n}$ is

$$
\begin{aligned}
& Q_{t_{n}}\left(W\left(t_{n}\right), A\left(t_{n}\right)\right) \\
& =\max _{\gamma_{n+1}, \ldots, \gamma_{N-1}} \mathrm{E}_{t_{n}}\left[e^{-r_{n, N}\left(T-t_{n}\right)} \max \left(W\left(T^{-}\right), C\left(A\left(T^{-}\right)\right)\right)+\sum_{j=n+1}^{N-1} e^{-r_{n, j}\left(t_{j}-t_{n}\right)} C\left(\gamma_{j}\right)\right],
\end{aligned}
$$

and the today's value of the annuity policy corresponds to $Q_{0}(W(0), A(0))$ which is a function of policy fee $\alpha$. Here, $\gamma_{1}, \ldots, \gamma_{N-1}$ are the control variables chosen to maximize the expected value of discounted cashflows, and expectation $\mathrm{E}_{t}[\cdot]$ is taken under the risk-neutral process conditional on $W_{t}$ and $A_{t}$. The fair fee $\alpha=\alpha^{*}$ corresponds to $Q_{0}(W(0), A(0))=W(0)$. It is important to note that control variables can be different for different realizations of underlying process and moreover the control variable $\gamma_{n}$ affects the transition law of the underlying wealth process from $t_{n}$ to $t_{n+1}$, i.e. calculating GMWB annuity price is solving optimal stochastic control problem. 


\section{Numerical valuation of GMWB}

In the case of continuous withdrawal, following the procedure of deriving the HamiltonJacobi-Bellman (HJB) equations in stochastic control problems, the value of the annuity under optimal withdrawal is found to be governed by a two-dimensional PDE; see Milevsky and Salisbury (2006), Dai et al. (2008) and Chen and Forsyth (2008). For discrete withdrawals, the governing PDE in the period between withdrawing dates is one-dimensional, similar to the Black-Scholes equation, with jump conditions at each withdrawing date to link the prices at the adjacent periods. Below we consider an alternative approach without dealing with PDEs.

The annuity price at any time $t$ for a fixed $A(t)$ is a function of $W$ only. Note $A\left(t_{n-1}^{+}\right)=A\left(t_{n}^{-}\right)=A$ is constant in the period $\left(t_{n-1}^{+}, t_{n}^{-}\right)$. Thus in a backward timestepping setting (similar to a finite difference scheme) the option price at time $t=t_{n-1}^{+}$ can be evaluated as the following expectation

$$
Q_{t_{n-1}^{+}}\left(W\left(t_{n-1}^{+}\right), A\right)=\mathrm{E}_{t_{n-1}}\left[e^{-r_{n} d t_{n}} Q_{t_{n}^{-}}\left(W\left(t_{n}^{-}\right), A\right) \mid W\left(t_{n-1}^{+}\right), A\right] .
$$

Assuming the conditional probability distribution density of $W\left(t_{n}^{-}\right)$given $W\left(t_{n-1}^{+}\right)$ is known as $p_{n}\left(w\left(t_{n}\right) \mid w\left(t_{n-1}\right)\right)$, then the above expectation can be evaluated by

$$
Q_{t_{n-1}^{+}}\left(W\left(t_{n-1}^{+}\right), A\right)=\int_{0}^{+\infty} e^{-r_{n} d t_{n}} p_{n}\left(w \mid W\left(t_{n-1}^{+}\right)\right) Q_{t_{n}^{-}}(w, A) d w .
$$

In the case of wealth process (4) the transition density $p_{n}\left(w\left(t_{n}\right) \mid w\left(t_{n-1}\right)\right)$ is known in closed form and we will use Gauss-Hermite quadrature for the evaluation of the above integration over an infinite domain. The required continuous function $Q_{t}(W, A)$ will be approximated by a cubic spline interpolation on a discretized grid in the $W$ space.

Any change of $A(t)$ only occurs at withdrawal dates. After the amount $\gamma_{n}$ is drawn at $t_{n}$, the wealth account reduces from $W\left(t_{n}^{-}\right)$to $W\left(t_{n}^{+}\right)=\max \left(W\left(t_{n}^{-}\right)-\gamma_{n}, 0\right)$, and the guarantee balance drops from $A\left(t_{n}^{-}\right)$to $A\left(t_{n}^{+}\right)=A\left(t_{n}^{-}\right)-\gamma_{n}$. Thus the jump condition of $Q_{t}(W, A)$ across $t_{n}$ is given by

$$
Q_{t_{n}^{-}}\left(W\left(t_{n}^{-}\right), A\left(t_{n}^{-}\right)\right)=\max _{0 \leq \gamma_{n} \leq A\left(t_{n}^{-}\right)}\left[Q_{t_{n}^{+}}\left(\max \left(W\left(t_{n}^{-}\right)-\gamma_{n}, 0\right), A\left(t_{n}^{-}\right)-\gamma_{n}\right)+C\left(\gamma_{n}\right)\right] .
$$

For optimal strategy, we chose a value for $\gamma_{n}$ under the restriction $0 \leq \gamma_{n} \leq A\left(t_{n}^{-}\right)$ to maximize the function value $Q_{t_{n}^{-}}(W, A)$ in (11). Repeatedly applying (10) and (11) backwards in time starting from

$$
Q_{t_{N}^{-}}\left(W\left(T^{-}\right), A\left(T^{-}\right)\right)=\max \left(W\left(T^{-}\right), C\left(A\left(T^{-}\right)\right)\right)
$$

gives us annuity value at $t=0$. 


\subsection{Overall algorithm description}

For a fixed guarantee balance $A$ between given withdrawal dates, the price $Q_{t}(W, A)$ can be numerically evaluated using (10). For now we leave details of computing (10) to the next section and assume it can be done with sufficient accuracy and efficiency. Starting from a final condition at $t=T^{-}$(just immediately before the final withdrawal), a backward time stepping using (10) gives solution up to time $t=t_{N-1}^{+}$. In order to apply the jump condition at each withdrawal date and find the solution $Q_{0}(W=$ $W(0), A=W(0)$ ), this backward time stepping needs to be done for many different levels of $A$. Applying jump condition (11) to the solution at $t=t_{N-1}^{+}$we obtain the solution at $t=t_{N-1}^{-}$from which further backward time stepping gives us solution at $t=t_{N-2}^{+}$, and so on. The numerical algorithm takes the following key steps:

- Step 1. Generate an auxiliary finite grid $0=A_{1}<A_{2}<A_{3} \cdots<A_{J}=W(0)$ to track the guarantee account $A$.

- Step 2. Discretize wealth account $W$ space as $W_{0}, W_{1}, \ldots, W_{M}$.

- Step 3. At $t=t_{N}=T$ apply the final condition at each node point $\left(W_{m}, A_{j}\right)$, $j=1,2, \ldots, J, m=1,2, \ldots, M$ to get payoff $Q_{T^{-}}(W, C(A))$.

- Step 4. Evaluate integration (10) for each of the $A_{j}$ to obtain $Q_{t_{N-1}^{+}}(W, A)$.

- Step 5. Apply the jump condition (11) to obtain $Q_{t_{N-1}^{-}}(W, A)$ for all possible jumps and find the jump to maximize $Q_{t_{N-1}^{-}}(W, A)$.

- Step 6. Repeat Step 4 and 5 for $t=t_{N-2}, t_{N-3}, \ldots, t_{1}$.

- Step 7. Evaluate integration (10) for the backward time step from $t_{1}$ to $t_{0}$ for the single node value $A=A_{J}=W(0)$ to obtain solution $Q_{0}\left(W, A_{J}\right)$ and take the value $Q_{0}\left(A_{J}, A_{J}\right)$ as the annuity price at $t=t_{0}$.

Below we discuss details of the algorithm of the numerical integration of (10) using Gauss-Hermite quadrature on a cubic spline interpolation, followed by the application of jump conditions.

\subsection{Numerical evaluation of the expectation}

Similar to a finite difference scheme, we propose to discretize the asset domain $\left[W_{\min }, W_{\max }\right]$ by $W_{\min }=W_{0}<W_{1}, \ldots, W_{M}=W_{\max }$, where $W_{\min }$ and $W_{\max }$ are the lower and upper boundary, respectively. For pricing GMWB, because of the finite reduction of $W$ at each withdrawal date, we have to consider the possibility of $W$ goes to zero, thus the lower 
bound $W_{\min }=0$. The upper bound is set sufficiently far from the spot asset value at time zero $W(0)$. A good choice of such a boundary could be $W_{\max }=W(0) \exp (5 \sigma \sqrt{T})$. The idea is to find option values at all these grid points at each time step from $t_{n}^{-}$to $t_{n-1}^{+}$through integration (101), starting at maturity $t=t_{N}^{-}=T^{-}$. At each time step we evaluate the integration (10) for every grid point by a high accuracy numerical quadrature.

At time step $t_{n}^{-} \rightarrow t_{n-1}^{+}$, the option value at $t=t_{n}^{-}$is known only at grid points $W_{m}, m=0,1, \ldots, M$. In order to approximate the continuous function $Q_{t}(W, A)$ from the values at the discrete grid points, we propose to use the cubic spline interpolation which is smooth in the first derivative and continuous in the second derivative. The error of cubic spline is $O\left(h^{4}\right)$ where $h$ is the size for the spacing of the interpolating variable, assuming a uniform spacing. The cubic spline interpolation involves solving a tri-diagonal system of linear equations for the second derivatives at all grid points. For a fixed grid and constant $r$ and $\sigma$, the tri-diagonal matrix can be inverted once and at each time step only the back-substitution in the cubic spline procedure is required.

The process for $W(t)$ between withdrawal dates is a standard stock market process given in (4), the conditional density of $W\left(t_{n}^{-}\right)$given $W\left(t_{n-1}^{+}\right)$is from a lognormal distribution, as is evident from (44). A more convenient and common practice is to work with $\ln (W)$ which is from normal distribution. Note that when we use $\ln (W)$, the minimum $W_{\min }$ cannot be zero, and instead we have to set $W_{\min }$ to be a very small value (e.g. $\left.W_{\min }=10^{-10}\right)$.

In order to make use of the highly efficient Gauss-Hermite numerical quadrature for integration over an infinite domain, we introduce a new variable

$$
Y\left(t_{n}\right)=\frac{\ln \left(W\left(t_{n}^{-}\right) / W\left(t_{n-1}^{+}\right)\right)-\nu_{n}}{\tau_{n}},
$$

where $\nu_{n}=\left(r_{n}-\alpha-\frac{1}{2} \sigma_{n}^{2}\right) d t_{n}$ and $\tau_{n}=\sigma_{n} \sqrt{d t_{n}}$ and denote the annuity price function $Q_{t}(w, \cdot)$ after this transformation as $Q_{t}^{(y)}(y, \cdot)$. Apparently from (44), $Y\left(t_{n}\right)$ is from standard normal distribution, thus by changing variable from $W\left(t_{n}\right)$ to $Y\left(t_{n}\right)$ the integration (10) becomes

$$
Q_{t_{n-1}^{+}}\left(W\left(t_{n-1}^{+}\right), A\right)=\frac{e^{-r_{n} d t_{n}}}{\sqrt{2 \pi}} \int_{-\infty}^{+\infty} e^{-\frac{1}{2} y^{2}} Q_{t_{n}^{-}}^{(y)}(y, A) d y,
$$

For an arbitrary function $f(x)$, the Gauss-Hermite quadrature is

$$
\int_{-\infty}^{+\infty} e^{-x^{2}} f(x) d x \approx \sum_{i=1}^{q} \lambda_{i}^{(q)} f\left(\xi_{i}^{(q)}\right)
$$

where $q$ is the order of the Hermite polynomial, $\xi_{i}^{(q)}$ are the roots of the Hermite polynomial $H_{q}(x)(i=1,2, \ldots, q)$, and the associated weights $\lambda_{i}^{(q)}$ are given by 


$$
\lambda_{i}^{(q)}=\frac{2^{q-1} q ! \sqrt{\pi}}{q^{2}\left[H_{q-1}\left(\xi_{i}^{(q)}\right)\right]^{2}} .
$$

In general, the abscissas and the weights for the Gauss-Hermite quadrature for a given order $q$ can be readily computed, e.g. using functions in Press et al. (1992); also as a reference, the abscissas and the weights for $q=5,6,16$ are presented in Luo and Shevchenko (2014).

Applying a change of variable $x=y / \sqrt{2}$ and use the Gauss-Hermite quadrature to (14), we obtain

$$
\begin{aligned}
\left.Q_{t_{n-1}^{+}}\left(W\left(t_{n-1}^{+}\right), A\right)\right) & =\frac{e^{-r_{n} d t_{n}}}{\sqrt{\pi}} \int_{-\infty}^{+\infty} e^{-x^{2}} Q_{t_{n}^{-}}^{(y)}(\sqrt{2} x, A) d x \\
& \approx \frac{e^{-r_{n} d t_{n}}}{\sqrt{\pi}} \sum_{i=1}^{q} \lambda_{i}^{(q)} Q_{t_{n}^{-}}^{(y)}\left(\sqrt{2} \xi_{i}^{(q)}, A\right) .
\end{aligned}
$$

If we apply the change of variable (13) and the Gauss-Hermite quadrature (16) to every grid point $W_{m}, m=0,1, \ldots, M$, i.e. let $W\left(t_{i-1}^{+}\right)=W_{m}$, then the option values at time $t=t_{i-1}^{+}$for all the grid points can be evaluated through (16).

As is commonly practiced in a finite difference setting for option pricing, the working domain in asset space is in terms of $X=\ln (W / W(0))$, where $W(0)$ is the spot value at time $t=0$. In our implementation we have $X_{\min }=\ln \left(W_{\min } / W(0)\right)$ and set $X_{\max }=$ $\ln \left(W_{\max } / W(0)\right)=5 \sigma \sqrt{T}$. The domain $\left(X_{\min }, X_{\max }\right)$ is uniformly discretised to yield the grid $\left(X_{\min }=X_{0}, X_{1}=\delta X, X_{2}=2 \delta X, \ldots, X_{M}=M \delta X=X_{\max }\right)$, where $\delta X=$ $\left(X_{\max }-X_{\min }\right) / M$. The grid point $W_{m}, m=0,1,2, \ldots, M$, is then given by $W_{m}=$ $W(0) \exp \left(X_{m}\right)$.

For each grid point $W_{m}$ or $X_{m}$, the variable $Y\left(t_{n}\right)$ is given by (13) with $W\left(t_{i-1}^{+}\right)=$ $W_{m}$, and the relationship between $X\left(t_{n}\right)=\ln \left(W\left(t_{n}\right) / W(0)\right)$ and $Y\left(t_{n}\right)$ for $W_{m}$ is worked out to be $X\left(t_{n}\right)=\tau_{n} Y\left(t_{n}\right)+\nu_{n}+X_{m}$, thus the numerical integration value for grid point $X_{m}$ at time $t_{n-1}^{+}$can be expressed, from (16), as

$$
Q_{t_{n-1}^{+}}^{(x)}\left(W\left(t_{n-1}^{+}\right), A\right) \approx \frac{e^{-r_{n} d t_{i}}}{\sqrt{\pi}} \sum_{i=1}^{q} \lambda_{i}^{(q)} Q_{t_{n}^{-}}^{(x)}\left(\sqrt{2} \tau \xi_{i}^{(q)}+\nu_{n}+X_{m}, A\right),
$$

where $Q_{t_{n}}^{(x)}\left(X\left(t_{n}\right), A\right)$ denotes the option value as a function of $X\left(t_{n}\right)=\ln \left(W\left(t_{n}\right) / W(0)\right)$. The above description of the numerical integration using Gauss-Hermite quadrature is illustrated in Figure 1,

The continuous function $Q_{t_{n}}^{(x)}(x, A)$ is approximated by the cubic spline interpolation based on variable $X$, given the values $Q_{t_{n}}^{(x)}\left(X_{m}, A\right)$ at discrete points $X_{m}, m=$ $0,1,2, \ldots, M$. The cubic spline interpolation has a much higher order of accuracy than linear or quadratic interpolation. Natural boundary conditions are imposed at the two 
ends $X_{0}=X_{\min }$ and $X_{M}=X_{\max }$, i.e. we assume zero second derivative of the spline function at the two ends.

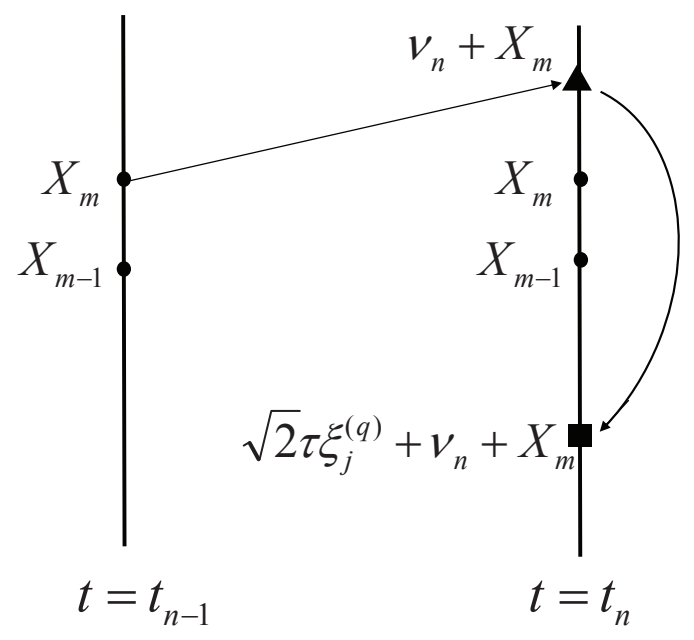

Figure 1: Illustration of Gauss-Hermite quadrature application for an arbitrary grid point $X_{m}$ at time $t=t_{n-1}$. The solid circles are fixed grid points, the solid triangle is the point of the expected mean at $t=t_{n}$ given $X_{m}$ at $t=t_{n-1}$, and the solid square is the $j-$ th quadrature point corresponding to $X_{m}$.

\subsection{Jump condition application}

Let us introduce an auxiliary finite grid $0=A_{1}<A_{2}<A_{3} \cdots<A_{J}=W(0)$ to track the remaining guarantee balance $A$, where $J$ is the total number of nodes in the guarantee balance amount coordinate. The upper limit $W(0)$ is needed because the remaining guarantee balance cannot exceed the target initial account value $W(0)$. For each $A_{j}$, we associate a continuous solution from (17) and the cubic spline interpolation. At every jump we let $A$ to be one of the grid points $A_{j}, 1 \leq j \leq J$. Since $A$ is always known at each jump to be one of the fixed nodal point values, there is no need to continuously track the actual evolution of the guarantee balance amount $A$ during the entire finite difference solving process. In addition, we also limit the number of possible discrete withdrawal amounts to be finite. The natural simple choice (though not necessary) is to only allow the guarantee balance to be equal to one of the grid points $0=A_{1}<A_{2}<A_{3} \cdots<A_{J}=W(0)$. This implies that, for a given balance $A_{j}$ at time $t_{n}^{-}$, the possible value after the withdraw at $t_{n}^{+}$has to be one of the grid 


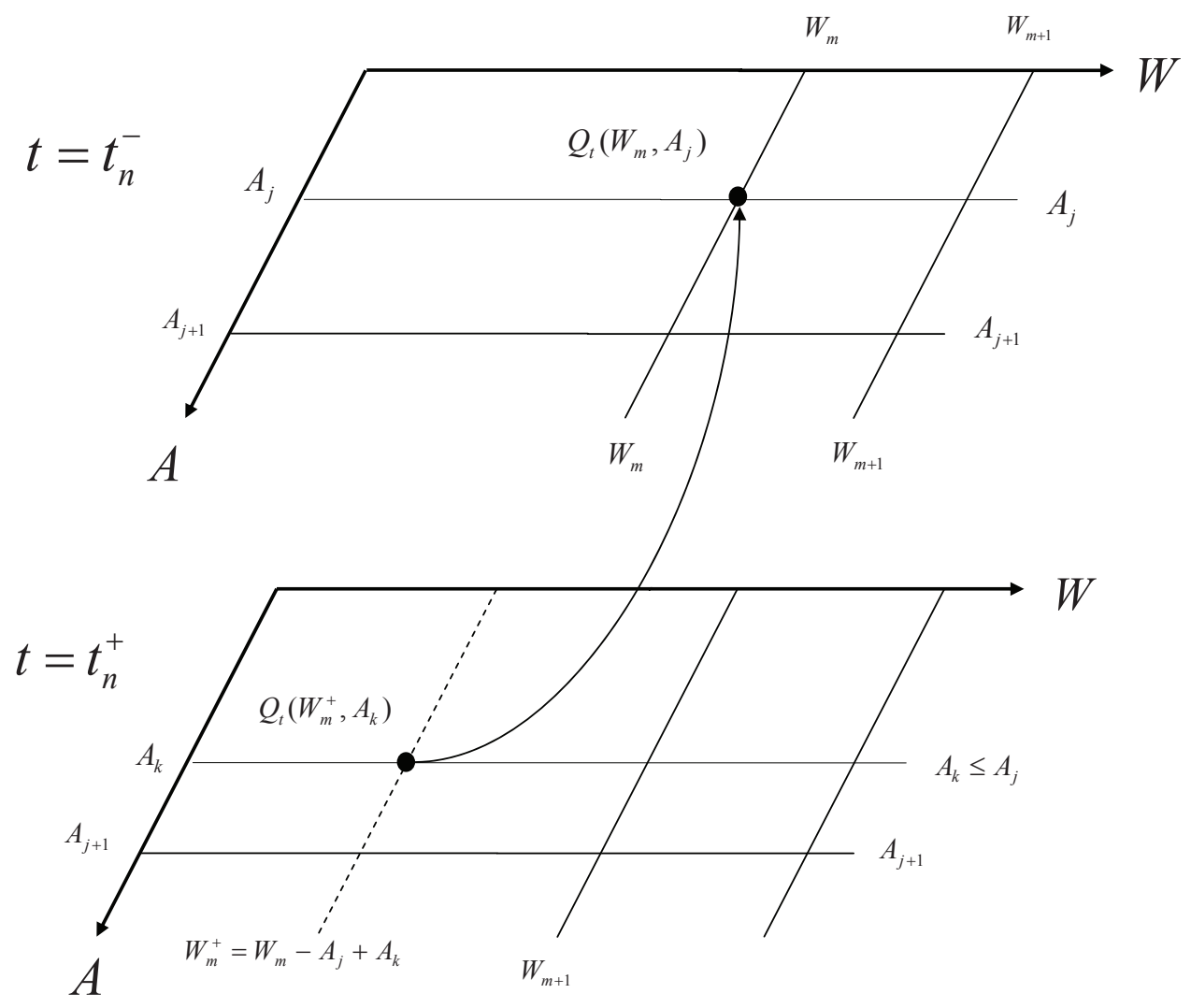

Figure 2: Illustration of jump conditions applied to finite difference grids.

points equal to or less than $A_{j}$, i.e. $A_{j}^{+}=A_{k}, 1 \leq k \leq j$. In other words, the withdraw amount $\gamma$ takes $j$ possible values: $\gamma=A_{j}-A_{k}, k=1,2, \ldots, j$.

Note the above restriction that $\gamma=A_{j}-A_{k}, k=1,2, \ldots, j$ is not necessary. The only real restriction is $\gamma \leq A_{j}$. However, without the restriction the value of $A_{j}^{+}$ after the jump falls between the grid points (not exactly on a grid point $A_{k}$ ) and a costly two-dimensional interpolation is required. The error due to this discretisation restriction can be easily reduced to acceptable level by increasing $J$.

For any $W=W_{m}, m=0,1, \ldots, M$ and $A=A_{j}, j=1, \ldots, J$, given that withdrawal amount can only take the pre-defined values $\gamma=A_{j}-A_{k}, k=1,2, \ldots, j$, irrespective of time $t_{n}$ and account value $W_{m}$, the jump condition (11) takes the following form for the specific numerical setting

$$
Q_{t_{n}^{-}}\left(W_{m}, A_{j}\right)=\max _{1 \leq k \leq j}\left[Q_{t_{n}^{+}}\left(\max \left(W_{m}-A_{j}+A_{k}, 0\right), A_{k}\right)+C\left(A_{j}-A_{k}\right)\right] .
$$

For optimal strategy, we chose a value for $1 \leq k \leq j$ to maximize the function value $Q_{t_{n}^{-}}\left(W_{m}, A_{j}\right)$ in (18). Note that although the jump amount $\gamma=A_{j}-A_{k}, k=1,2, \ldots, j$ 
is independent of time $t_{n}$ and account value $W_{m}$, the value $Q_{t_{n}^{+}}\left(\max \left(W_{m}-A_{j}+\right.\right.$ $\left.\left.A_{k}, 0\right), A_{k}\right)$ depends on all variables $\left(W_{m}, A_{j}, t_{n}\right)$ and the jump amount. Thus the above jump has to be performed for every node point $\left(W_{m}, A_{j}\right), 1 \leq m \leq M, 1 \leq j \leq J$ at every withdrawal date. Obviously for every node point $\left(W_{m}, A_{j}\right)$ we have to attempt $j$ jumps to find the maximum value for $Q_{t_{n}^{-}}\left(W_{m}, A_{j}\right)$.

When $W_{m}-A_{j}+A_{k}>0$, the value $Q_{t_{n}^{+}}\left(W_{m}-A_{j}+A_{k}, A_{k}\right)$ can be obtained by interpolation from the values at the $M$ discrete grid points. Overall we have $J$ numerical solutions (obtained through integration) to track, corresponding to each of the $A_{j}$ value, $1 \leq j \leq J$. Figure 2 illustrates the application of the jump condition.

In (18), to obtain $Q_{t_{n}^{+}}\left(W_{m}-A_{j}+A_{k}, A_{k}\right)$ from solution $Q_{t_{n}^{+}}\left(W, A_{k}\right)$, only a onedimensional interpolation is required, since the coordinate in the guarantee balance space $A$ remain the same at $A_{k}$. Essentially we have given $M+1$ values at $W_{0}, W_{1}, \ldots, W_{M}$ to find the value at $W=W_{m}-A_{j}+A_{k}$. For this purpose we chose the same cubic spline interpolation as used in approximating the continuous function $Q_{t}(W, \cdot)$ in Section 3.2 . As shown in a convergence study by Forsyth et al. (2002), it is possible for a PDE based numerical algorithm for discretely sampled path-dependent option pricing to be non-convergent (or convergent to an incorrect answer) if the interpolation scheme is selected inappropriately. All the previous studies of numerical PDE solution for path dependent (Asian or lookback options) used either a linear or a quadratic interpolation in applying the jump conditions. In our experience a better choice is the cubic spline interpolation (Press et al. (1992)).

Remarks It is worth pointing out that part of the good efficiency of the present algorithm for pricing GMWB under rational policyholder behavior is due to the fact that the same cubic spline interpolation is used for both numerical integration (10) and the application of jump condition (18). A clear advantage of the present numerical algorithm over PDE based finite difference approach is that significantly smaller number of time steps are required by the present method. In fact the number of time steps needed by the proposed method is the same as the number of withdrawal dates, i.e. there is no need to sub-divide the time period between two consecutive withdrawal dates into finer time steps - a single step is sufficient because the transition density over the finite time period in (10) is exact and there is no approximation error due to finite time steps. On the other hand, in general the finite difference method requires dividing the period between two consecutive withdrawal dates into finer time steps for a good accuracy due to the finite difference approximation to the partial derivatives. The above comment also applies to other derivatives such as the American option with discrete exercise dates, Asian options and Target Accumulation Redemption Notes, etc. with discrete 
payment dates. The accuracy of a central difference finite difference scheme is second order both in time and space. Here the error due to finite number of grid points in $W$ space is from cubic spline interpolation and the Gaussian-Hermite quadrature, while in finite difference method the error is from the finite difference approximation to the space derivatives. Both errors can be reduced by increasing the number of grid points in $W$ space (reducing grid size).

\subsection{An alternative - GHQC with moment matching}

In calculation of annuity price expectation (14), the probability density for $Y\left(t_{n}\right)$ is known in closed form. In general the closed form pdf may not be known, and here we propose a moment matching to replace (14), i.e. assuming we do not know the density in closed form but we know the moments of the distribution, we can still use the GHQC algorithm by matching the numerically integrated moments with the known moments. Let $p(y)$ denote the unknown density of $Y\left(t_{n}\right)$, then the integration in (14) becomes

$$
Q_{t_{n-1}^{+}}\left(W\left(t_{n-1}\right), A\right)=e^{-r_{n} d t_{n}} \int_{-\infty}^{+\infty} p(y) Q_{t_{n}^{-}}^{(y)}(y, A) d y
$$

which can be re-written as

$$
Q_{t_{n-1}^{+}}\left(W\left(t_{n-1}\right), A\right)=e^{-r_{n} d t_{n}} \int_{-\infty}^{+\infty} e^{-y^{2}} \times\left[e^{y^{2}} p(y)\right] Q_{t_{n}^{-}}^{(y)}(y, A) d y
$$

Applying Gauss-Hermite quadrature (15) to (20) we then have

$$
Q_{t_{n-1}^{+}}\left(W\left(t_{n-1}\right), A\right) \approx \sum_{i=1}^{q} \lambda_{i}^{(q)} \widetilde{p}\left(\xi_{i}^{(q)}\right) Q_{t_{n}^{-}}^{(y)}\left(\xi_{i}^{(q)}, A\right)
$$

where the function $\widetilde{p}(y)=e^{y^{2}} p(y)$ which is also unknown. Defining a new weight $\omega_{i}^{(q)}=\lambda_{i}^{(q)} \widetilde{p}\left(\xi_{i}^{(q)}\right)$, the numerical quadrature for the integration simplifies to

$$
\int_{-\infty}^{+\infty} p(y) Q_{t_{n}^{-}}^{(y)}(y, A) d y \approx \sum_{i=1}^{q} \omega_{i}^{(q)} Q_{t_{n}^{-}}^{(y)}\left(\xi_{i}^{(q)}, A\right)
$$

Now we proceed to find the unknown coefficients $\omega_{i}^{(q)}, i=1,2, \ldots, n$ by matching moments. Recognizing that if we replace $Q_{t_{n}^{-}}^{(y)}(y, A)$ by $y^{K}$, the integration yields the $K$-th moment corresponding to the pdf $p(y)$, thus

$$
\mathrm{E}_{t_{n-1}}\left[Y\left(t_{n}\right)^{K}\right]=\int_{-\infty}^{+\infty} p(y) y^{K} d y=M_{K}(y) \approx \sum_{i=1}^{q} \omega_{i}^{(q)}\left(\xi_{i}^{(q)}\right)^{K},
$$

where $M_{K}(y)$ denotes the $K$-th moment of random variable $y$. If we let $K=0,1, \ldots, n-$ 1 we then have $n$ equations to determine the $n$ unknown coefficients $\omega_{i}^{(q)}, i=1,2, \ldots, q$. 
In our GMWB evaluation framework the annuity value is a function of $X\left(t_{n}\right)=$ $\ln \left(W\left(t_{n}\right) / W(0)\right)$, and for each node point $X_{m}$ we have $X\left(t_{n}\right)=\tau_{n} Y\left(t_{n}\right)+\nu_{n}+X_{m}$. To match the central moment for random variable $X\left(t_{n}\right)$ (centered at $\nu_{n}+X_{m}$ ), equation (23) becomes

$$
\begin{aligned}
\mathrm{E}_{t_{n-1}}\left[\left(X\left(t_{n}\right)-\nu_{n}-X_{m}\right)^{K}\right] & =\int_{-\infty}^{+\infty} p_{X\left(t_{n}\right)}(x)\left(x-\nu_{n}-X_{m}\right)^{K} d x \\
& \approx \sum_{i=1}^{q} \omega_{i}^{(q)}\left(\tau \xi_{i}^{(q)}\right)^{K}, \quad K=0,1, \ldots, q-1,
\end{aligned}
$$

where $p_{X\left(t_{n}\right)}(x)$ is the pdf for random variable $X\left(t_{n}\right)$. For the standard stock market process, the central moments for $X\left(t_{n}\right)$ are simply

$$
\mathrm{E}_{t_{n-1}}\left[\left(X\left(t_{n}\right)-\nu_{n}-X_{m}\right)^{K}\right]= \begin{cases}0, & \text { if } K \text { is odd } \\ \tau^{K}(K-1) ! !, & \text { if } K \text { is even }\end{cases}
$$

where $(K-1)$ !! is the double factorial, that is, the product of every odd number from $K-1$ to 1 .

Remarks Although in (24) the Gauss-Hermite weights do not appear explicitly, it is still a direct application of the full Gauss-Hermite quadrature. To make this clear, we can substitute back $\omega_{i}^{(q)}=\lambda_{i}^{(q)} \widetilde{p}\left(\xi_{i}^{(q)}\right)$ in (24) to obtain a system of linear equations for the unknown function values $\widetilde{p}\left(\xi_{i}^{(q)}\right), i=0,1, \ldots, q-1$

$$
\mathrm{E}_{t_{n-1}}\left[\left(X\left(t_{n}\right)-\nu_{n}-X_{m}\right)^{K}\right] \approx \sum_{i=1}^{q} \lambda_{i}^{(q)} \widetilde{p}\left(\xi_{i}^{(q)}\right)\left(\tau \xi_{i}^{(q)}\right)^{K}, \quad K=1, \ldots, q,
$$

and obviously solving (25) is equivalent to solving (24).

The direct application of the Gauss-Hermite quadrature is most suitable for the standard normal distribution, that is why the derivation of (24) is through the variable $y$. In general if the distribution of $X$ is not a normal distribution, we can still define $y$ through the mean and standard deviation for conditional probability of the underlying (conditional on the value given at time step $i-1$ ).

Having found the $n$ coefficients $\omega_{i}^{(q)}$ by solving the system of linear equations (24), the expected option value $Q_{t_{n-1}^{+}}\left(W\left(t_{n-1}^{+}\right), A\left(t_{n-1}^{+}\right)\right)$is then approximated as

$$
Q_{t_{n-1}^{+}}\left(W\left(t_{i-1}^{+}\right), A\left(t_{i-1}^{+}\right)\right) \approx e^{-r_{n} d t_{n}} \sum_{i=1}^{q} \omega_{i}^{(q)} Q_{t_{n}^{-}}^{x}\left(\tau_{n} \xi_{i}^{(q)}+\nu_{n}+X_{m}\right) .
$$

The GHQC algorithm with moment matching is exactly the same as the one described earlier, except now we have (26) instead of (17) for the numerical quadrature, and an extra step to solve the system of linear equations (24) for the new weights to match moments. For convenience we denote the above moment matching algorithm as GHQC-M. 


\section{Numerical Results}

Below we present numerical results for pricing GMWB with static and optimal policyholder strategies and compare with Monte Carlo and finite difference methods when appropriate.

\subsection{GMWB pricing results}

In general the price for a variable GMWB annuity is a function of $\left(\alpha, r, \sigma, \beta, g, W(0), N_{w}\right)$. In practice, the policyholder is charged exactly the amount of the initial investment $W(0)$. In other words, there is no additional charge on the policyholder. This is possible only because the issuer can set the "right" amount of the fee $\alpha$ (which is "continuously" taken from the investment account), so that the annuity price $V$ equals to the initial investment $W(0)$. Thus the pricing problem becomes: giving $\left(r, \sigma, \beta, g, W(0), N_{w}\right)$, finding the correct fee $\alpha$ so that the annuity price $V=W(0)$. Obviously this is an iterative process: one starts with an initial guess for the fee and compute the annuity price, and repeats the pricing a few times while iteratively adjust the fee value.

If the withdraw amount at each withdrawal date is predetermined at the beginning of the contract, then the behavior of the policyholder is called "static". In this case the paths of account $W$ can be simulated and a standard Monte Carlo simulation method can be used to price the GMWB. On the other hand if the policyholder optimally decide the amount of withdraw at each withdrawal date, then the behavior of the policyholder is called "dynamic". Below we show results for both static and dynamic cases. The static case allows a comparison between Monte Carlo and GHQC, further validating the new algorithm.

We have also implemented an efficient finite difference (FD) algorithm for pricing variable GMWB both with static and dynamic policyholder behaviors. In what follows results from GHQC will be compared with those from FD. In all the literature reviewed in the Introduction, only Dai et al. (2008) and Chen and Forsyth (2008) have presented some results for the price or fair fee of GMWB under the dynamic (optimal)

policyholder behavior, both studies have used a finite difference method. We will also compare GHQC results with their FD results.

\subsubsection{GMWB fair fees with static policyholder behavior}

In a static case the withdrawal amount is pre-determined for each withdrawal date. In this case there is no need for GHQC or FD to track many solutions for multiple levels of the guarantee account $A$ - only one solution is required and at each payment date the jump condition applies to the single solution (therefore no need for a grid in 
guarantee account $A$ ). Since the withdrawal amount is known at every payment date, the stochastic paths of the underlying $W$ can be simulated by Monte Carlo. Table 1 shows results of the fair fee for the static case calculated using GHQC, GHQC-M, FD and MC. The withdrawal frequency is quarterly (four times per year), i.e. $N_{w}=4$. The interest rate is $r=5 \%$ and volatility is $\sigma=20 \%$. The unit of the fees (continuous rate) shown in all the following Tables is in basis point (bp) which is $0.01 \%$, i.e. a 100 basis points is $1 \%$.

For GHQC, GHQC-M and FD the number of grids for $W$ is set at $M=400$. The number of time steps for FD is set at 100 per year. The number of quadrature points for GHQC and GHQC-M is set at $q=9$. Unless otherwise stated, the above numerical inputs were used in all the following examples in this paper. We have also used $q=16$ for the number of quadrature points and found the results in the fair fee are identical at least in the first four digits. We observe that for two values of the fair fee from different methods to be identical in the first four digits, it requires the values of GMWB price with the same inputs to be identical in the first 6 digits. In the Monte Carlo calculations we used $2 \times 10^{7}$ simulated paths (including antithetic paths).

In Table 1 the numbers in the parentheses (the last column) are the estimated "standard errors" of the MC estimate for the fair fee. Note that the fair fee cannot be directly simulated by MC, it is inversely calculated in an iterative process as described earlier, thus the standard error of the fair fee cannot be directly estimated from MC samples of prices given a fee. Here we estimated the standard error by the difference in fees due to the standard errors in the price by the following procedure.

Let $\widehat{Q}$ and $\epsilon_{Q}$ be the MC estimate of GMWB price and its standard error respectively. The fair fee estimator $\widehat{\alpha}^{*}$ is obtained through $\widehat{Q}\left(\widehat{\alpha}^{*}\right)=W(0)$. An upper bound for the fair fee $\widehat{\alpha}_{U}^{*}$ can be estimated from $\widehat{Q}\left(\widehat{\alpha}_{U}^{*}\right)+\epsilon_{Q}=W(0)$, and a lower bound $\widehat{\alpha}_{L}^{*}$ can be estimated from $\widehat{Q}\left(\widehat{\alpha}_{L}^{*}\right)-\epsilon_{Q}=W(0)$. Having obtained the lower and upper bounds for the fair fee corresponding to the standard error in the price, we then estimate the standard error of the fair fee by $\epsilon_{\alpha}=\left(\widehat{\alpha}_{U}^{*}-\widehat{\alpha}_{L}^{*}\right) / 2$. One can also took an alternative approach: numerically calculate the derivative $\frac{\partial \alpha}{\partial Q}$ and estimate the error in the fair fee by $\epsilon_{\alpha}=\left|\frac{\partial \alpha}{\partial Q}\right| \times \epsilon_{Q}$. We found the two approaches give us virtually the same answers. For example, at $g=10 \%$, the first approach gives $\epsilon_{\alpha}=0.155$, while the second method yields $\epsilon_{\alpha}=0.154$. In calculating $\frac{\partial \alpha}{\partial Q}$, we perturb the fair fee by $1 \%$ on each side of the $\mathrm{MC}$ estimated fair fee value and compute the corresponding price changes.

As shown in Table 1, the fair fee is an increasing function of the contractual withdrawal rate. The GHQC and GHQC-M produced identical results for all the withdrawal rates, at least for all the first 4 digits shown. Between GHQC and FD, the maximum absolute difference in the fee is $0.1 \mathrm{bp}$ which occurs at the highest contractual rate 


\begin{tabular}{cccccc}
\hline contractual rate, $g$ & maturity $T=1 / g$ & GHQC, bp & GHQC-M, bp & FD, bp & MC, bp \\
\hline $4 \%$ & 25.00 & 17.69 & 17.69 & 17.79 & $17.23(0.120)$ \\
$5 \%$ & 20.00 & 28.33 & 28.33 & 28.30 & $28.29(0.125)$ \\
$6 \%$ & 16.67 & 40.33 & 40.33 & 40.31 & $40.37(0.130)$ \\
$7 \%$ & 14.29 & 53.31 & 53.31 & 53.28 & $53.20(0.135)$ \\
$8 \%$ & 12.50 & 66.99 & 66.99 & 66.93 & $67.02(0.145)$ \\
$9 \%$ & 11.11 & 81.23 & 81.23 & 81.21 & $81.23(0.145)$ \\
$10 \%$ & 10.00 & 95.81 & 95.81 & 95.78 & $95.79(0.155)$ \\
$15 \%$ & 6.67 & 171.9 & 171.9 & 171.8 & $171.5(0.185)$ \\
\hline
\end{tabular}

Table 1: Fair fee $\alpha$ in bp $(1 \mathrm{bp}=0.01 \%)$ as a function of annual contractual rate $g$ for the static case. The parameters for the annuity product are $r=5 \%, \sigma=20 \%, N_{w}=4$ (quarterly withdrawal frequency).

$g=15 \%$. A difference of 0.1 basis point in the fee $\alpha$ is about 1 cent per year for a $\$ 1000$ account. Between GHQC and MC, the maximum absolute difference in the fee is $1.2 \mathrm{bp}$, about 12 cents a year for a $\$ 1000$ account, which also occurs at the highest contractual rate $g=15 \%$. The computing time requirement for both FD and HGQC in the static case is very fast - both took a fraction of a second to compute a single price. We will have a detailed comparison of the computing speed in the next section dealing with pricing GMWB in the dynamic case, where the computation is much more demanding because multiple solutions of many levels of guarantee amount have to be tracked and multiple jumps have to be applied for finding the optimal strategy.

\subsubsection{GMWB fair fees with optimal policyholder behavior}

Table 2 shows results of the fair fee for the dynamic case at a quarterly withdrawal frequency $\left(N_{w}=4\right)$, calculated using GHQC and FD. In this example the number of grids in the guarantee account $A$ is $J=100$ for both GHQC and FD. The maximum difference between GHQC and FD in the calculated fair fee is $0.15 \mathrm{bp}$, less than 2 cents a year for a $\$ 1000$ account, which occurs at the lowest contractual rate $g=4 \%$. Again the GHQC-M results (not shown in the table) were identical to those from GHQC in at least the first four digits shown.

Compared with the static case, the fees for the dynamic case are much higher the dynamic fee is $15 \%$ higher than the static fee at the contract rate $g=15 \%$ and this difference increases to $217 \%$ at $g=4 \%$. Recall that the maturity $T=1 / g$, so the lowest rate corresponds to the longest maturity and vise versa. With a fixed withdrawal frequency, a longer maturity means more opportunities for the policyholder to make optimal decisions to maximize the total cashflow from holding the variable GMWB 


\begin{tabular}{cccc}
\hline contractual rate, $g$ & maturity $T=1 / g$ & GHQC, bp & FD, bp \\
\hline $4 \%$ & 25.00 & 56.09 & 55.94 \\
$5 \%$ & 20.00 & 70.06 & 69.96 \\
$6 \%$ & 16.67 & 83.73 & 83.64 \\
$7 \%$ & 14.29 & 97.11 & 97.03 \\
$8 \%$ & 12.50 & 110.3 & 110.2 \\
$9 \%$ & 11.11 & 123.2 & 123.1 \\
$10 \%$ & 10.00 & 136.0 & 135.9 \\
$15 \%$ & 6.67 & 199.0 & 199.0 \\
\hline
\end{tabular}

Table 2: Fair fee $\alpha$ in bp $(1 \mathrm{bp}=0.01 \%)$ as a function of annual contractual rate $g$ for the dynamic case with a quarterly withdrawal frequency $\left(N_{w}=4\right)$. The other parameters for the GMWB product are $r=5 \%, \sigma=20 \%, \beta=10 \%$.

contract. Thus at the lowest contract rate the fee for the dynamic case shows the highest percentage increase from the static case, highlighting the value of optimal decisions under uncertainty.

\begin{tabular}{ccccc}
\hline contractual rate, $g$ & maturity $T=1 / g$ & GHQC, bp & FD, bp & Dai et al. (2008), bp \\
\hline $4 \%$ & 25.00 & 56.77 & 56.68 & 56 \\
$5 \%$ & 20.00 & 70.92 & 70.78 & 69 \\
$6 \%$ & 16.67 & 84.76 & 84.63 & 83 \\
$7 \%$ & 14.29 & 98.30 & 98.18 & 97 \\
$8 \%$ & 12.50 & 111.6 & 111.5 & 111 \\
$9 \%$ & 11.11 & 124.7 & 124.6 & 124 \\
$10 \%$ & 10.00 & 137.7 & 137.5 & 137 \\
$15 \%$ & 6.67 & 201.7 & 201.6 & 198 \\
\hline
\end{tabular}

Table 3: Fair fee $\alpha$ in bp $(1 \mathrm{bp}=0.01 \%)$ as a function of annual contractual rate $g$ for the dynamic case with a monthly withdrawal frequency $\left(N_{w}=12\right)$. The other parameters for the GMWB product are $r=5 \%, \sigma=20 \%, \beta=10 \%$.

Table 3 shows results of the fair fee for the dynamic case at a monthly withdrawal frequency $\left(N_{w}=12\right)$ calculated using GHQC and FD. For these monthly withdrawal cases the number of grids in $A$ was set at $A=300$ for both GHQC and FD. The maximum difference between GHQC and FD in the calculated fair fees shown in Table 3 is 0.2 basis point, about 2 cents a year for a $\$ 1000$ account. Comparing Table 3 and Table 2, our results from both GHQC and FD consistently show a higher fee for a higher withdraw frequency at all contractual rates. In general a higher withdrawal frequency should have a higher fair fee than a lower withdrawal frequency, since the former has 
a higher annuity value for the same fee. The higher withdrawal frequency allows the policyholder to have more opportunities to make optimal decisions to maximize the total cashflow and so it is more valuable. Nevertheless the relative difference in fees between monthly and quarterly withdrawal contracts is in the order of $1 \%$ only. In absolute terms the maximum difference in the fees between the monthly and quarterly withdrawal contracts is less than 3 basis points, occurring at the highest contractual rate $g=15 \%$. A difference of 3 basis points in fees is only about 30 cents per year for a $\$ 1000$ account value. The close agreement in fees (or prices) between the quarterly and monthly withdrawal frequency indicates that the results for the monthly withdrawal frequency should already be approaching those of the continuous case.

Also shown in Table 3 are the results for a continuous withdrawal model obtained by Dai et al. (2008) solving a two dimensional linear complementary problem using a penalty finite difference method. In the case of a continuous withdrawal model, the policyholder can make a decision at any instance of time and the withdraw amount can be infinitely small or finite, thus it can be more optimal than any discrete case. However, our results for the discrete withdrawal model at a monthly frequency are slightly higher than those of Dai et al. (2008) for the continuous case, which should not happen if the numerical calculations for both the discrete model and the continuous model are exact. Still, as can be calculated from Table 3, the difference in the fees between our monthly withdrawal model and the continuous model is small - the maximum absolute difference is 3.7 basis points occurring at the highest contractual rate $g=15 \%$.

\begin{tabular}{cccc}
\hline contractual rate, $\mathrm{g}$ & maturity $\mathrm{T}=1 / \mathrm{g}$ & $\mathrm{GHQC}$ & $\mathrm{FD}$ \\
\hline $4 \%$ & 25.00 & 102.0 & 101.3 \\
$5 \%$ & 20.00 & 123.6 & 123.2 \\
$6 \%$ & 16.67 & 144.1 & 143.7 \\
$7 \%$ & 14.29 & 163.5 & 163.2 \\
$8 \%$ & 12.50 & 182.1 & 181.8 \\
$9 \%$ & 11.11 & 199.8 & 199.6 \\
$10 \%$ & 10.00 & 216.9 & 216.7 \\
$15 \%$ & 6.67 & 293.8 & 293.8 \\
\hline
\end{tabular}

Table 4: Fair fee $\alpha$ as a function of annual guarantee rate $g$ for the dynamic case with a quarterly withdrawal frequency $\left(N_{w}=4\right)$ and with the penalty rate reduced to $\beta=5 \%$. The other parameters for the GMWB product are $r=5 \%, \sigma=20 \%$.

Table 4 shows results for a quarterly withdrawal contract when the penalty rate $\beta$ is reduced to $5 \%$. As a result of the reduced penalty rate, the fair fees increase very significantly: at $g=15 \%$ (shortest maturity) the fee is increased by $45 \%$ and this 


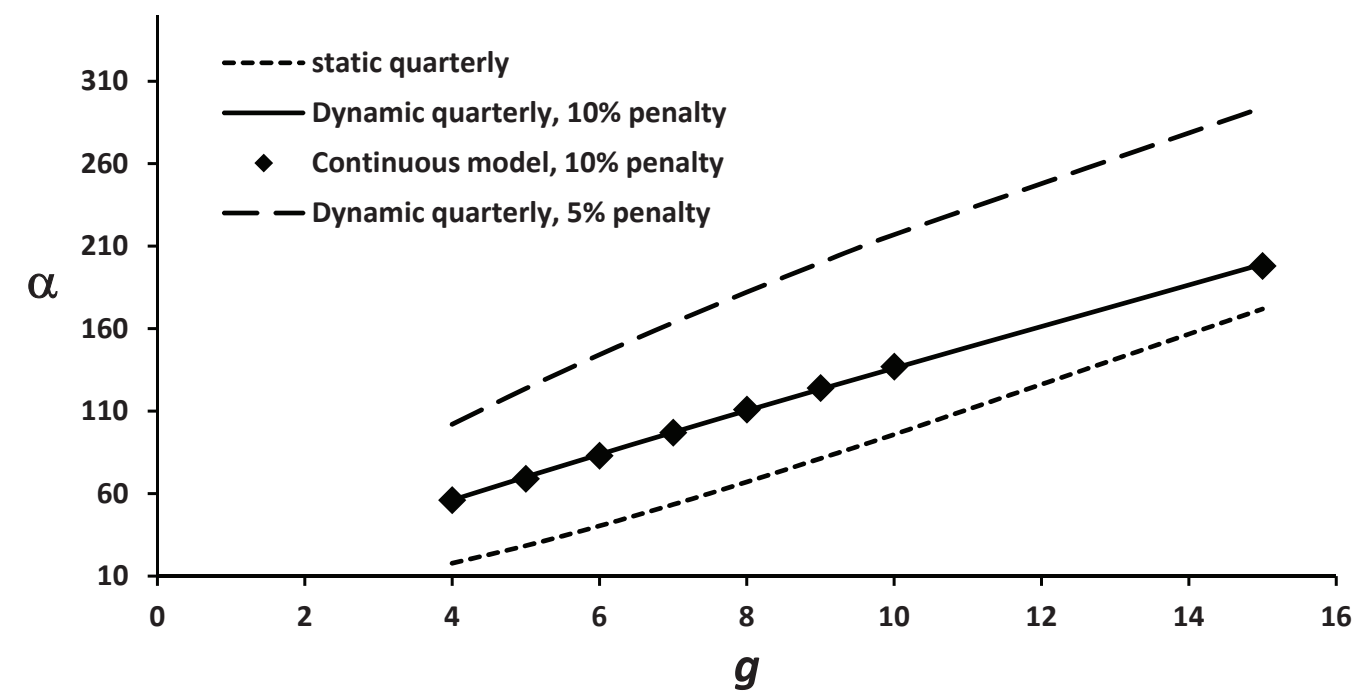

Figure 3: Fair fee $\alpha$ as a function of annual guarantee rate $g$ for three discrete withdrawal contracts at a quarterly withdraw rate, in comparison with the continuous model.

increase becomes almost $80 \%$ at $g=4 \%$ (the longest maturity). This demonstrates that the optimal decision is much more valuable when it is not restricted by a penalty. To illustrate the relative magnitudes of the fees for various cases, Figure 3 compares the fair fees for four cases: static quarterly withdrawal, dynamic quarterly withdrawal with $10 \%$ penalty charge, dynamic quarterly withdrawal with $5 \%$ penalty charge, and the continuous withdrawal model calculated by Dai et al. (2008). In Figure 3 data for all the three curves of the discrete withdrawal model are calculated using the GHQC algorithm. Results of FD and GHQC-M for the same cases are not shown because they appear identical on the graph.

To compare computing speed between FD and GHQC, we record the CPU time for a single price calculation - the calculation of the fair fee involves many such calculations in an iterative process. The CPU used for all the calculations in this study is Intel(R) Core(TM) i5-2400 @3.1GHz. Apart from the numerical mesh size and time steps, the CPU time for a single price calculation for a dynamic case also depends on the maturity and withdrawal frequency. Longer maturity and higher frequency demand more computing time. Among the examples shown in Table 2 and Table 3 , the case with a quarterly withdraw frequency at the shorted maturity $(g=15 \%$ and $T=1 / g=6.67$ years) is the least demanding in computing time. For this case the FD took 14 seconds and the GHQC took 2 seconds to compute a single price. On the other end, the case 
with a monthly withdraw frequency at the longest maturity $(g=4 \%$ and $T=1 / g=25$ years) is the most demanding in computing time, and for this case the FD took 482 seconds and the GHQC took 167 seconds to compute a single price. As discussed earlier, both FD and GHQC used the same grids for both $A$ and $W$. Obviously the speed advantage of GHQC is more pronounced at lower withdrawal frequency - only a single time step is required for GHQC between consecutive withdrawal dates, while for FD the time step size has to be sufficiently small for good accuracy, irrespective of the withdrawal frequency. For example, to price a GMWB at a yearly withdrawal frequency, the GHQC is more than 15 times as fast as FD using the same grids in $W$ and $A$. Note that we believe our finite difference implementation for pricing GMWB is already very efficient - for example the tri-diagonal matrix for the linear equation discretising the PDE is constructed and inverted only once for all the constant time steps and for all the solutions at all levels of the guarantee amount $A$, taking advantage of the constant interest rate and volatility. So each time step in the FD only involves a simple back-substitution which takes little CPU time.

\begin{tabular}{cccc}
\hline frequency & volatility & Chen and Forsvth (2008) & GHQC \\
\hline yearly & 0.2 & 129.1 & 129.1 \\
half-yearly & 0.2 & 133.5 & 133.7 \\
yearly & 0.3 & 293.3 & 293.5 \\
half-yearly & 0.3 & 302.4 & 302.7 \\
\hline
\end{tabular}

Table 5: Comparison of fair fee $\alpha$ between results of GHQC and those from finite difference by Chen and Forsyth (2008). The input parameters are $g=10 \%, \beta=10 \%$, $r=5 \%$.

In Chen and Forsyth (2008), the fair fees for the discrete withdrawal model with $g=10 \%$ for the yearly $(N w=1)$ and half-yearly $(N w=2)$ withdrawal frequency at $\sigma=0.2$ and $\sigma=0.3$ were presented in a carefully performed convergence study, with the same values for other input parameters $(r=5 \%, \beta=10 \%)$. Table 5 compares GHQC results with those of Chen and Forsyth (2008). The values of Chen and Forsyth (2008) quoted in Table 5 correspond to their finest mesh grids and time steps at $M=2049$ for $W, J=1601$ for $A$ and $N=1920$ for $t$, while our GHQC values were obtained using $M=400, J=100$ and with $n=9$ for the number of quadrature points. As shown in Table 5, the maximum absolute difference in the fair fee rate between the two numerical studies is only $0.3 \mathrm{bp}$, and the average absolute difference of the four cases in the table is less than $0.2 \mathrm{bp}$. In relative terms, the maximum difference is less than $0.15 \%$, and the average magnitude of the relative differences between the two studies 
is less than $0.08 \%$. Chen and Forsyth (2008) did not provide CPU numbers for their calculations of fair fees. In our case each calculation of the fair fee in Table 5, which involves a Newton iteration, took about 5 seconds.

\section{Conclusion}

In this paper we have presented a new algorithm for pricing variable annuities with GMWB features under both static and dynamic (optimal) policyholder behaviors. The new method is neither based on solving PDEs using finite difference nor on simulations using Monte Carlo. The new algorithm relies on numerically computing the expected option values in a backward time-stepping between withdrawal dates through GaussHermite integration quadrature applied on a cubic spline interpolation, either using transition density or matching moments. At discrete withdrawing dates the proper jump conditions are applied at various withdrawal account levels which allows the optimal withdrawal decision to be made.

Numerical results from the new algorithm for a series of GMWB contract are presented, in comparison with results using finite difference method. The comparison demonstrates that the new algorithm produces results very close to those of the finite difference (from our own or from literature), but at the same time it is significantly faster. Using the same grids for the space variables for the underlying and the guarantee amount, the relative difference in price between GHQC and FD is in the order of $0.001 \%$, and the absolute difference in the fair fee rate between the two methods is in the order of $0.1 \mathrm{bp}$, which is one cent per year in a $\$ 1000$ account.

So far we have assumed the policyholder will always live beyond the maturity date or there is always someone there to make optimal withdrawal decisions for the entire duration of the contract. In reality an elderly policyholder may die before maturity date, especially for a contract with a long maturity. For example, according to the Australian Life Table, a male aged 60 will have more than $57 \%$ probability to die before the age of 85 . So for a 60 year old male taking up a GMWB contract with a maturity of 25 years $(g=4 \%)$, it is unrealistic not to consider the probability of death during the contract period and design and pricing the suitable contract accordingly. It is not difficult to consider adding some death benefits on top of GMWB, i.e. combining GMWB with some kind of life insurance. The new method will enable us to efficiently explore possibilities of new products considering both market process and death process. Further work includes admitting other stochastic risk factors such as stochastic interest rate or volatility. It remains to be seen whether the new GHQC algorithm can still be significantly faster than the finite difference method in higher dimensions for 
solving the optimal stochastic control problem arising from pricing GMWB under the dynamic policyholder behavior.

\section{References}

Bacinello, A., P. Millossovich, A. Olivieri, and E. Pitacco (2011). Variable annuities: a unifying valuation approach. Insurance: Mathematics and Economics 49(1), 285297.

Bauer, D., A. Kling, and J. Russ (2008). A universal pricing framework for guaranteed minimum benefits in variable annuities. ASTIN Bulletin 38(2), 621-651.

Chen, Z. and P. Forsyth (2008). A numerical scheme for the impulse control formulation for pricing variable annuities with a guaranteed minimum withdrawal benefit (gmwb). Numerische Mathematik 109(4), 535-569.

Dai, M., K. Y. Kwok, and J. Zong (2008). Guaranteed minimum withdrawal benefit in variable annuities. Mathematical Finance 18(4), 595-611.

Forsyth, P. A., K. R. Vetzal, and R. Zvan (2002). Convergence of numerical methods for valuing path-dependent options using interpolation. Review of Derivatives Research 5, 273-314.

Longstaff, F. and E. Schwartz (2001). Valuing American options by simulation: a simple lieast-squares approach. Review of Financial Studies 14, 113-147.

Luo, X. and P. V. Shevchenko (2014). Fast and simple method for pricing exotic options using gauss-hermite quadrature on a cubic spline interpolation. To appear in Journal of Financial Engineering.

Milevsky, M. A. and T. S. Salisbury (2006). Financial valuation of guaranteed minimum withdrawal benefits. Insurance: Mathematics and Economics 38(1), 21-38.

Press, W. H., S. A. Teukolsky, W. T. Vetterling, and B. P. Flannery (1992). Numerical Recipes in $C$. Cambridge University Press.

United Nations (2013). World Population Prospects: The 2012 Revision, Highlights and Advance Tables. United Nations, New York: United Nations, Department of Economic and Social Affairs, Population Division. 